\title{
Les contributions majeures de Michel Balinski dans le vote et le choix social
}

Rida Laraki*

\author{
MAJOR CONTRIBUTIONS OF MICHEL BALINSKI IN VOTING \\ AND SOCIAL CHOICE THEORY
}

Michel Balinski a vécu et travaillé principalement aux États-Unis et en France. Né à Genève en 1933, sa famille s'installe aux États-Unis en 1940. Il y fait ses études et, après un doctorat à Princeton, occupe des postes de professeur en mathématiques, économie et sciences administratives dans les universités de Princeton, de Pennsylvanie, de la ville de New York, de Yale et de Stony Brook (État de New York). De retour en France en 1980, il est nommé directeur de recherche « de classe exceptionnelle » au CNRS à l'École polytechnique où il fut directeur du Laboratoire d'économétrie jusqu'à sa retraite en 1999. Il est décédé à Bayonne le 4 février 2019.

Michel Balinski est le fondateur et le premier rédacteur en chef de la prestigieuse revue internationale Mathematical Programming, il est également l'un des fondateurs de la Mathematical Optimization Society, qu'il a présidée. Ses recherches sur la programmation linéaire et non linéaire, la programmation en nombres entiers, les polyèdres convexes, les correspondances sur les graphes, les mariages stables et le vote ont remporté de nombreux prix internationaux, dont le prix renommé John von Neumann Theory en 2013.

Michel Balinski se distingue en tant qu'inventeur exceptionnel de plusieurs systèmes équitables de vote et de représentation. Il a développé - seul ou en collaboration - des théories complètes pour plusieurs problèmes distincts et a caractérisé les mécanismes permettant de les résoudre. Il a su rendre accessibles ses recherches pour un grand public, ce qui explique en partie l'impact pratique de ses travaux qui peuvent être classés en trois thèmes:

- comment répartir les sièges entre les régions ou les partis;

- comment répartir les sièges entre les régions et les partis;

- comment élire un(e) candidat(e) ou classer plusieurs candidats.

* CNRS (Lamsade, Dauphine-PSL) et Université de Liverpool. Correspondance : Lamsade, Université Paris Dauphine, 1 place du Maréchal de Lattre de Tassigny, 75016 Paris, France. Courriel : rida.laraki@dauphine.fr 


\section{RÉPARTIR LES SIĖGES ENTRE LES RÉGIONS OU LES PARTIS}

La première trace de l'intérêt de Michel Balinski pour la représentation se trouve dans son article de 1965 sur la programmation en nombres entiers (Balinski [1965]) (lauréat du prix Lanchester d'INFORMS). Dans ce document, il formule le problème de répartition « équitable » des sièges de la Chambre des représentants (ou de toute assemblée) entre les différents États (régions, sections) en fonction de leur population.

Dans une série d'articles avec H. Peyton Young - dont l'un (Balinski et Young [1975b]) a reçu le prix Lester R. Ford de la Mathematical Association of America (MAA) en 1975 -, ils établissent une théorie complète de la répartition (voir l'ouvrage Balinski et Young [1982]). Ils caractérisent les seules méthodes qui évitent les diverses anomalies possibles - tels le «paradoxe de l'Alabama » ou le « nouvel État ». Ils élaborent un théorème d'impossibilité et montrent de manière concluante qu'une méthode, celle de Webster, répond le mieux à tous les critères d'une bonne répartition. En particulier, la méthode de Webster est considérée comme la seule méthode acceptable qui traite de manière impartiale les États peu peuplés par rapport aux plus peuplés. Leurs résultats n'ont pas entraîné de changement de méthode aux États-Unis, mais leur ouvrage a eu une application pratique directe dans d'autres pays, notamment au Royaume-Uni. Le livre est généralement considéré comme la solution définitive sur la répartition et a reçu le prix George H. Hallett 2008 de l'American Political Science Association « pour un livre publié il y a au moins dix ans et qui a apporté une contribution durable à $[. .$.$] la représentation et aux systèmes électoraux ». Il constitue une référence$ majeure dans un arrêt de la Cour suprême (ministère du Commerce vs Montana, 503 US 442 [1992]). Cela a conduit le Royaume-Uni à adopter la méthode de Webster dans sa loi de 2011 sur le système de vote auquel Michel Balinski a participé (Balinski et al. [2010]).

\section{RÉPARTIR DES SIÈGES ENTRE LES RÉGIONS ET LES PARTIS}

Les méthodes de vote aux Pays-Bas et au Mexique ont motivé la deuxième contribution majeure de Michel Balinski dans le domaine des systèmes électoraux. L'intention de ces méthodes est de donner à chacune des régions (États, cantons ou districts) leur juste part des sièges et d'assurer simultanément à chaque parti politique sa juste part des sièges sur la base du nombre total de ses voix dans toutes les régions. C'est le problème de la répartition " matricielle ». Dans une série d'articles, commençant en 1987 avec Gabrielle Demange puis seul ou en collaboration, il a développé une théorie complète et une méthode de « répartition biproportionnelle » (Balinski [1987], [2006]; Balinski, Aït-Sahalia et Demange [1988], [1990]; Balinski et Demange [1989a], [1989a]) et a montré comment l'appliquer à divers contextes (Balinski et Ramírez González [1996], [1997a], [1997b], [1999]; Balinski [2002], [2004]; Balinski et Pukelsheim [2006]), notamment pour éviter le gerrymandering (Balinski [2008a]). Ce dernier article a obtenu le prix Lester R. Ford de la MAA en 2008. 
Ce mécanisme est maintenant utilisé pour répartir les parlements de plusieurs villes et cantons de Suisse : la ville et le canton de Zurich depuis 2006, le canton de Schaffhouse depuis 2008, le canton d'Argovie depuis 2009, le canton de Zoug depuis 2014, le canton de Nidwald depuis 2014. À Zoug, il a été adopté par référendum, obtenant $81 \%$ des voix contre le statu quo. A Nidwald, il a également été adopté par référendum, obtenant $57 \%$ des voix sur un ensemble de cinq possibilités. Dans ces deux cas, les grands partis politiques ont activement fait campagne contre.

\section{ÉLIRE ET CLASSER DES CANDIDATS}

Pendant plus d'un demi-siècle, le théorème d'impossibilité d'Arrow a dominé la théorie du vote et la théorie du choix social. Il montre qu'il n'existe pas de méthode satisfaisante pour concilier les différents ordres de préférence d'un électorat ou jury: quatre propriétés simples, apparemment naturelles et peu contraignantes, ne peuvent pas être réunies ensemble.

Les travaux récents que Michel Balinski et moi avons menés conjointement apportent une solution à l'impossibilité d'Arrow, inspirés par la pratique (vin, patinage artistique, gymnastique, concours de musique, entre autres). La théorie du jugement majoritaire est développée dans plusieurs articles publiés à partir de 2007, et plus principalement dans l'ouvrage publié aux MIT Press en 2011 (Balinski et Laraki [2011]).

Dans la nouvelle formulation du problème, les électeurs - à l'instar des universitaires et des juges de jury de vin - évaluent leurs concurrents dans un « langage commun » de mentions (par exemple : inacceptable, faible, bon, très bon). Une reformulation est essentielle pour deux raisons. La première est que le modèle traditionnel conduit à des paradoxes (ceux de Condorcet et d'Arrow). La seconde est que diverses expériences démontrent que les électeurs ne sont pas disposés à exprimer une préférence stricte pour un ou plusieurs candidats. Il est démontré que le jugement majoritaire est le seul dans une famille de modes de scrutin qui évite les paradoxes d'Arrow et de Condorcet et qui neutralise au mieux le vote stratégique.

Depuis lors, le jugement majoritaire est utilisé pour élire et ranger: sélectionner les étudiants pour des bourses à Dauphine, attribuer un prix de journalisme à Harvard, élire des membres de l'Académie des sciences en Angleterre, choisir le nom d'un laboratoire de recherche à l'université Paris Diderot, classer des candidats pour un poste universitaire à Montpellier, à l'École polytechnique, à Santiago ou encore aux Arts et Métiers, mais aussi pour élire une candidate à l'élection présidentielle française de 2017 par LaPrimaire.org, avec plus de 35000 participants (LaPrimaire.org [2016]). Enfin, Terra Nova (Terra Nova [2011]) et un projet de Constitution pour l'Europe (European Constitution.eu [2018]) soutiennent le système, et le Mouvement Génération.s, nouveau mouvement politique français, utilise le jugement majoritaire dans son processus de décision interne (Mouvement Génération.s [2018]). En 2018, l'association Mieux Voter a été créée pour le promouvoir (Mieux Voter [2018]). 


\section{MON MICHEL}

Je dois beaucoup à Michel. Il m'a accepté en 1996, après mes études à l'École polytechnique, dans le DEA « Optimisation, jeux et modélisation en économie », qu'il avait cofondé et dirigé. Il m'a fortement soutenu pour intégrer le CNRS en 2001 après ma thèse sous la direction de Sylvain Sorin. Il m'a initié au vote en m'invitant avec deux autres collègues de l'École polytechnique à participer à la première expérience électorale sur le terrain, au Monde (Balinski et al. [2003]). Dans le cadre de cette expérience organisée en 2002 à Orsay, des électeurs ont été invités, le jour de l'élection, à expérimenter le vote par assentiment ; le faire essayer à grande échelle en parallèle d'un scrutin présidentiel est une idée de Michel qui date de 1995, quand il avait proposé de le faire avec Laurent Mann, thésard qui venait de terminer son doctorat sur cette méthode. Michel a été - encore une fois - un grand visionnaire, car depuis, à chaque élection présidentielle en France et ailleurs, des équipes expérimentent des modes de scrutin alternatifs.

Michel Balinski, en plus d'être un chercheur exceptionnel, était un collègue intègre, un homme humaniste, profond et sophistiqué, un directeur hors norme aimé et respecté par ses collègues et secrétaires, et pour moi, un ami fidèle et attentif, un confident, un conseiller, et parfois même, un père. Il me manquera énormément.

\section{RÉFÉRENCES BIBLIOGRAPHIQUES}

\section{Ouvrages sur le vote}

BALINSKi M. [2004], Le suffrage universel inachevé, Paris, Belin.

BALINSKi M. et LARAKI R. [2011], Majority Judgment: Measuring, Ranking, and Electing, Cambridge (Mass.), The MIT Press.

Balinski M. et Young H. P. [1982], Fair Representation: Meeting the Ideal of One Man, One Vote, New Haven, Yale University Press; $2^{\mathrm{e}}$ éd.: Washington (D. C.), Brookings Institution Press, 2001 ; trad. jap. : Tokyo, Chikura-Shobo Publishing Company, 1987.

\section{Articles sur le vote}

BALINSKi M. [1965], «Integer Programming: Methods, Uses, Computation », Management Science, 12 (3), p. 253-313; réédité dans G. B. Dantzig et A. F. Veinott, JR. (dir.) [1968], Mathematics of the Decision Sciences: Lectures in Applied Mathematics, Providence, American Mathematical Society, vol. 11, p. 179-256,; dans H. W. KuHN (dir.) [1970], Proceedings of the Princeton Symposium on Mathematical Programming, Princeton, Princeton University Press, p. 199-266; dans M. Jünger et al. (dir.) [2010], 50 Years of Integer Programming, 1958-2008, Berlin, Springer, p. 133-197. (Article lauréat du prix Frederick W. Lanchester de l'INFORMS en 1965.)

BALINSKi M. [1987], « Notes on Defining Proportionality between Matrices », présentation lors du $8^{\mathrm{e}}$ Mathematical Programming Symposium, Chugoku Electric Power Co., Hiroshima, 5-6 novembre.

Balinski M. [1996], « How Should Data Be Rounded? », dans L. Rüschendorf, B. SCHWEIZER et M. D. TAYLOR (dir.), Distributions with Fixed Marginals and Related Topics, Hayward, Institute of Mathematical Statistics, p. 33-44. 
BALINSKi M. [2002], « Une "dose" de proportionnelle: le système électoral mexicain », Pour la science, avril, p. 58-59; trad. all.: « Verhältniswahlrecht häppchenweise », Spektrum der Wissenschaft, octobre 2002, p. 72-74; réédité dans le dossier « Fairness, Kooperation, Demokratie: Die Mathematik des Sozialverhaltens », Spektrum der Wissenschaft, 5, 2006, p. 19-21.

Balinski M. [2006], « Apportionment: Uni- and Bi-dimensional », dans B. Simeone et F. Pukelsheim (dir.), Mathematics and Democracy: Recent Advances in Voting Systems and Collective Choice, Berlin, Springer, p. 43-53.

BALINSKi M. [2008a], « Fair Majority Voting (or How to Eliminate Gerrymandering) », American Mathematical Monthly, 115 (2), p. 97-113. (Article lauréat du prix Lester R. Ford de la Mathematical Association of America.)

BaLINSKi M. [2008b], « The Majority Judgement: A New Theory for Better Decisions », IFORS News, décembre, p. 5-6.

BALINSKi M. [2010], «The Majority Judgment: A New Mechanism for Electing and Ranking ", dans Beyond the Ballot Box, Thimphou, The Center for Bhutan Studies, p. 257-268.

Balinski M. [2016], " How Majority Voting Betrayed Voters Again in 2016 », The Conversation, $1^{\text {er }}$ décembre, https://theconversation.com/how-majority-voting-betrayed-voters-again-in-2016-69206.

BALINSKI M. [2018], « Réponse à des critiques du jugement majoritaire », CREST Working Paper, 2018-10.

Balinski M., AïtT-Sahalia Y. et Demange G. [1988], « Le redressement des tables de contingence: deux nouvelles approches », dans J.-C. Milleron (dir.), Mélanges économiques. Essais en l'honneur de Edmond Malinvaud, Paris, Economica, p. 897- 914.

Balinski M., Aït-Sahalia Y. et Demange G. [1990], « The Adjustment of Contingency Tables: Two New Approaches », dans P. Champsaur (dir.), Essays in Honor of Edmond Malinvaud, Cambridge (Mass.), The MIT Press, vol. 3, p. 99-114.

Balinski M. et Demange G. [1989a], «Algorithms for Proportional Matrices in Reals and Integers ", Mathematical Programming, 45 (1-3), p. 193-210.

Balinski M. et Demange G. [1989b], « An Axiomatic Approach to Proportionality Between Matrices », Mathematics of Operations Research, 14 (4), p. 700-719.

BaLInSKi M., Jennings A. et LaRaKi R. [2009], « Monotonic Incompatibility between Electing and Ranking », Economics Letters, 105 (2), p. 145-147.

Balinski M., Johnston R., McLean I. et Young P., avec l'aide de Cummine A. [2010], Drawing a New Constituency Map for the United Kingdom: The Parliamentary Voting System and Constituencies Bill 2010, Londres, The British Academy.

BALINSKI M. et LARAKI R. [2007a], "A Theory of Measuring, Electing and Ranking », PNAS, 104 (21), p. 8720-8725.

BALINSKI M. et LARAKI R. [2007b], « Le jugement majoritaire: l'expérience d'Orsay », Commentaire, 118, été, p. 413-419.

BALINSKi M. et LARAKI R. [2011], « Election by Majority Judgment: Experimental Evidence », dans B. DoleZ, B. Grofman, et A. Laurent (dir.), In Situ and Laboratory Experiments on Electoral Law Reform: French Presidential Elections, Berlin, Springer, p. 13-54.

BALINSKI M. et LARAKI R. [2012a], « Jugement majoritaire versus vote majoritaire (via les présidentielles 2011-2012) », Revue française d'économie, 27, p. 11-44.

BALINSKI M. et LARAKI R. [2012b], « Le jugement majoritaire: une nouvelle théorie du vote », séminaire au Collège de France, 29 février.

Balinski M. et Laraki R. [2013], " How Best to Rank Wines », dans O. Güvenen, H. Serbat, E. Giraud-Héraud et M.-C. Pichery (dir.), Wine Economics: Quantitative Studies and Empirical Applications, Basingstoke, Palgrave Macmillan, p. 149-172.

Balinski M. et Laraki R. [2014a], « Judge: Don't Vote! », Operations Research, 62 (3), p. 483-511.

Balinski M. et LaRaKi R. [2014b], "What Should "Majority Decision" Mean? », dans J. Elster et S. NovaK (dir.), Majority Decisions: Principles and Practices, Cambridge, Cambridge University Press, p. 103-131. 
BALINSKI M. et LARAKI R. [2016a], « Pour éviter un nouveau 21 avril, instaurons le "jugement majoritaire" ", The Conversation, 21 avril, http://theconversation.com/fr; rééd. : Commentaire, 154, été, p. 413-415.

Balinski M. et LARAKI R. [2016b], « Trump and Clinton Victorious: Proof that US Voting System Doesn't Work », The Conversation, 9 mai, https://theconversation.com/trumpand-clinton-victorious-proof-that-us-voting-system-doesnt-work-58752.

Balinski M., LARAKi R., LASLier J.-F. et VAN DER Straeten K. [2003], « Le vote par assentiment: une expérience », Cahier du Laboratoire d'économétrie de l'École polytechnique, 2003-13.

Balinski M. et Pukelsheim F. [2006], " Matrices and Politics », dans E. P. Liski, J. Isotalo, S. Puntanen et G. P. H. Styan (dir.), Festschrift for Tarmo Pukkila on his 60th Birthday, Tampere, University of Tampere, p. 233-242.

Balinski M. et Pukelsheim F. [2007], « Die Mathematik der Deppelten Gerechtigkeit », Spektrum der Wissenschaft, avril, p. 76-80.

Balinski M. et Rachev S. T. [1993], "Rounding Proportions: Rules of Rounding », Numerical Functional Analysis and Optimization, 14 (5-6), p. 475-501.

Balinski M. et Rachev S. T. [1997], « Rounding Proportions: Methods of Rounding », The Mathematical Scientist, 22, p. 1-26.

Balinski M. et Ramírez GonZÁLez V. [1996], « A Case Study of Electoral Manipulation: The Mexican Laws of 1989 and 1994 », Electoral Studies, 15 (2), p. 203-217.

BALINSKi M. et RAMíRez GonzÁLEZ V. [1997a], « Los sistemas electorales de 1989-90 y 1994 para la elección de diputados en México: Análisis y crítica », Estudios Sociológicos, 15, p. 137-185.

BALINSKi M. et RAmÍREZ GonZÁLEZ V. [1997b], « Mexican Electoral Law: 1996 Version », Electoral Studies, 16 (3), p. 329-340.

Balinski M. et Ramírez González V. [1999], « Mexico’s 1997 Apportionment Defies its Electoral Law », Electoral Studies, 18 (1), p. 117-124.

Balinski M. et Shahidi N. [1998], «A Simple Approach to the Product Variation Problem via Axiomatics », Operations Research Letters, 22 (4), p. 129-135.

BaLINSKi M. et Young H. P. [1975a], «Apportionment Schemes and the Quota Method», American Mathematical Monthly, 84 (6), p. 450-455.

Balinski M. et Young H. P. [1975b], « The Quota Method of Apportionment », American Mathematical Monthly, 82 (7), p. 701-730. (Article lauréat du prix Lester R. Ford de la Mathematical Association of America.)

Balinski M. et Young H. P. [1977], « On Huntington Methods of Apportionment », SIAM Journal on Applied Mathematics, 33 (4), p. 607-618.

Balinski M. et Young H. P. [1978a], « Stability, Coalitions and Schisms in Proportional Representation Systems », American Political Science Review, 72 (3), p. 848-858.

BaLINSKi M. et Young H. P. [1978b], « The Jefferson Method of Apportionment », SIAM Review, 20 (2), p. 278-284.

Balinski M. et Young H. P. [1979a], « Criteria for Proportional Representation », Operations Research, 27 (1), p. 80-95.

Balinski M. et Young H. P. [1979b], «Quotatone Apportionment Methods », Mathematics of Operations Research, 4 (1), p. 31-38.

BaLINSKi M. et Young H. P. [1980], « The Webster Method of Apportionment », PNAS, 77 (1), p. 1-4.

Balinski M. et Young H. P. [1981], « Parliamentary Representation and the Amalgam Method », Canadian Journal of Political Science, 14 (4), p. 797-812.

Balinski M. et Young H. P. [1982], « Fair Representation in the European Parliament », Journal of Common Market Studies, 20 (4), p. 361-373.

Balinski M. et Young H. P. [1983a], " Apportioning the United States House of Representatives », Interfaces, 13 (4), p. 35-43.

Balinski M. et Young H. P. [1983b], « Fair Electoral Distribution », Policy Options/ Options Politiques, 4, juillet-août, p. 30-32. 
Balinski M. et Young H. P. [1985], " The Apportionment of Representation », dans H. P. Young (dir.), Proceedings of Symposia in Applied Mathematics. Vol. 33: Fair Allocation, Providence, American Mathematical Society, p. 1-29.

Balinski M. et Young H. P. [1994], "Apportionment ", dans S. M. Pollock, M. H. RothкоpF et A. BARnetT (dir.), Handbooks in Operations Research and Management Science. Vol. 6: Operations Research and the Public Sector, Amsterdam, North-Holland, p. 529-560.

EUROPEAN CONSTITUTION.Eu [2018], « Federal Institutions in Brief », https://europeanconstitution.eu/federal-institutions-in-brief.

LAPrimaire.org [2016], « Résultats du 2nd tour de LaPrimaire.org », 31 décembre, https://articles.laprimaire.org/résultats-du-2nd-tour-de-laprimaire-org-2d61b2ad1394.

Mieux Voter [2018], " Choisir - Élire - Décider, avec le jugement majoritaire », https://mieuxvoter.fr.

MouvEMENT GÉNÉRATION.s [2018], « Les statuts de Génération.s, une histoire singulière vers la démocratie ", https://www.generation-s.fr/comite/mouvement-generation-s/ article/les-statuts-de-generation-s-une-histoire-singuliere-vers-la-democratie.

Terra Nova M. [2011], « Rendre les élections aux électeurs : le jugement majoritaire », http://tnova.fr. 